\title{
Molecular detection of canine bufaviruses in wild canids
}

\author{
Irene Melegari ${ }^{1} \cdot$ Federica Di Profio $^{1}$ - Andrea Palombieri ${ }^{1}$. Vittorio Sarchese ${ }^{1}$. Georgia Diakoudi ${ }^{2}$. Serena Robetto ${ }^{3}$. \\ Riccardo Orusa ${ }^{3} \cdot$ Fulvio Marsilio $^{1} \cdot$ Kristián Bányai $^{4} \cdot$ Vito Martella $^{2} \cdot$ Barbara Di Martino $^{1}$ (])
}

Received: 29 March 2019 / Accepted: 3 May 2019 / Published online: 6 June 2019

(c) Springer-Verlag GmbH Austria, part of Springer Nature 2019

\begin{abstract}
Novel protoparvoviruses genetically related to human and non-human primate bufaviruses (BuVs) have been detected recently in respiratory and enteric specimens collected from dogs and cats. In this study, by molecular screening of archival collections of faecal samples from wolves and foxes, we detected BuVs with a rate of $17.1 \%(7 / 41)$ and $10.5 \%$ (9/86), respectively. Sequence analysis of a portion of the ORF2 gene region of nine positive samples showed that the viruses in these samples were closely related to $\mathrm{BuVs}$ (97.5-99.0\% nucleotide sequence identity) found in domestic carnivores.
\end{abstract}

\section{Introduction}

Protoparvoviruses are small $(20-25 \mathrm{~nm})$, nonenveloped viruses with a single-stranded DNA genome of 4.5-5.5 kb flanked by two terminal hairpin structures $[1,2]$ and represent a distinct genus in the family Parvoviridae. The genus Protoparvovirus includes the species Carnivore protoparvovirus 1 (canine parvovirus type 2 or CPV-2, feline panleukopenia parvovirus or FPV, racoon parvovirus, mink enteritis virus), Eulipotyphila protoparvovirus 1 (megabat bufavirus 1), Primate protoparvovirus 1 (human bufavirus 1a), Primate protoparvovirus 2 (rhesus parvovirus 1), Primate protoparvovirus 3 (human cutavirus), Rodent protoparvovirus 1 (minute virus of mice), Rodent protoparvovirus 2 and 3 (rat parvoviruses), Ungulate protoparvovirus 1, Ungulate protoparvovirus 2 (porcine parvoviruses), and Chiropteran protoparovirus 1 (https://talk.ictvonline.org/ictv-reports/

Handling Editor: William G Dundon.

Barbara Di Martino

bdimartino@unite.it

1 Faculty of Veterinary Medicine, Università degli Studi di Teramo, Piazza Aldo Moro, 45, 64100 Teramo, Italy

2 Faculty of Veterinary Medicine, Università Aldo Moro di Bari, Valenzano, Italy

3 Istituto Zooprofilattico Sperimentale del Piemonte, della Liguria e della Valle d'Aosta, Torino, Italy

4 Institute for Veterinary Medical Research, Centre for Agricultural Research, Hungarian Academy of Sciences, Budapest, Hungary ictv_online_report/ssdnaviruses/w/parvoviridae/1045/genus -protoparvovirus/history).

Novel protoparvoviruses were identified recently during an outbreak of canine infectious respiratory disease (CIRD) that occurred in a litter of mixed-breed 5-month-old puppies in Italy [3]. Also, by screening collections of faecal and respiratory specimens obtained from juvenile dogs, the virus was detected in stool samples of dogs with or without enteric disease and in nasal and oropharyngeal swabs of dogs with CIRD. Analysis of the complete genome sequences of three strains (ITA/2015/297, HUN/2012/126 and HUN/212/22) [3] showed that a novel canine parvoviruses, designated canine bufaviruses ( $\mathrm{CBuVs})$, exhibited the highest sequence the identity in NS1 $(66.9 \%-69.4 \%$ nucleotide [nt] and $47.2 \%-51.4 \%$ amino acid [aa]) and VP1 (66\%-68.2\% nt and $62.5 \%-67.2 \%$ aa) regions to human and non-human primate bufaviruses (BuVs) (Primate protoparvovirus 1 and Primate protoparvovirus 2) [4-6], while identity to the well-known CPV-2 (Carnivore protoparvovirus 1 ) was $56 \% \mathrm{nt}$ and $40.6 \%$ aa in NS1 and $42.6 \%$ nt and $33.4 \%$ aa in VP1. According to the criteria established by the International Committee on Taxonomy of Viruses (ICTV) for classification of parvoviruses into the same species (cutoff $>85 \%$ aa sequence identity in the NS1 protein), the canine protoparvovirus may be considered a member of a new species within the genus Protoparvovirus, for which he name "Carnivore protoparvovirus 2" is proposed.

More recently, evidence was obtained by molecular screening of respiratory specimens collected from cats with or without signs of upper respiratory tract disease (URTD) and stool samples from diarrhoeic animals that feline hosts 
are also susceptible to similar viruses [7]. Sequence analysis of the nearly complete VP2 coding region of three feline strains (ITA/2012/TE109, ITA/2015/BA509 and ITA/2017/ BA291) revealed that the viruses displayed $>99.9 \% \mathrm{nt}$ sequence identity to $\mathrm{CBuVs}$ detected in dogs [3]. These findings seem to indicate that genetically closely related parvoviruses circulate between dogs and cats, suggesting limited host-species restriction among domestic carnivores. In order to obtain a more complete picture of the ecology of this novel protoparvovirus, we investigated whether wild canids can also harbor this virus given the availability in our laboratory of two sample collections of enteric origin from wolves (Canis lupus) and red foxes (Vulpes vulpes).

A total of 127 rectal swabs were collected between September 2009 and April 2017 by the National Reference Center for Wild Animal Diseases (CeRMAS) and submitted to the Faculty of Veterinary Medicine, University of Teramo, Italy. The faecal panel consisted of 41 samples from necropsized wolves found dead after traumatic events and 86 samples from red foxes shot during regular hunting seasons. Viral nucleic acids were extracted using a QIAamp UltraSens Virus Kit (QIAGEN GmbH, Hilden, Germany), following the manufacturer's protocol, and stored at $-80^{\circ} \mathrm{C}$ until use.

The presence of $\mathrm{CBuV}$ DNA was assessed by real-time PCR (q-PCR) as described previously [3]. Viral DNA quantification was performed using TaqMan Fast Advanced master mix (Thermo Fisher Scientific, USA) in a $25-\mu l$ volume comprising $5 \mu \mathrm{l}$ of extracted DNA and $20 \mu \mathrm{l}$ of master mix. The primers CPPV-L3-For (5'-TGAACAAGAAATAGA CAACATTGTCAT-3') and CPPV-L3-Rev (5'-AAAGAG CAGTTAGGTCATTGTTGT-3') and TaqMan probe CPPVL3 (5'-Fam-CCAAACAAGGTACAGGACAGGAAGAAA CAACACAA-BHQ1-3') were used at concentrations of 200 and $100 \mathrm{nM}$, respectively. The DNA copy number was calculated based on a standard curve that was generated using a series of tenfold dilutions of standard plasmid vector $\mathrm{pCR}$ 2.1 (TA Cloning Kit, Invitrogen, Ltd, Milan, Italy) containing a 98-bp fragment of the VP2 region of the canine protoparvovirus 2 strain ITA/2011/297-15 (GenBank accession no. MF198244) [3]. To minimize potential contamination, the plasmid was prepared in a separate room, and microtubes containing plasmid standards were not taken into the PCR setup room. Three negative controls were included in each run. Viral DNA was detected in 7 out of $41(17.1 \%)$ faecal samples collected from wolves and in 9 out of 86 (10.5\%) samples obtained from foxes. Viral loads ranged from $3.6 \times 10^{1}$ to $5.3 \times 10^{4} \mathrm{DNA}$ copies $/ 5 \mu \mathrm{l}$ of template (mean, $5.4 \times 10^{3}$ DNA copies $/ 5 \mu$ l, cutoff cycle threshold, 39.00). Using the specific primers CPPV $165 \mathrm{~F}$ (5'-CTGGTT TAATCCAGCAGACT-3') and CPPV 371R (5'-TGAAGA CCAAGGTAGTAGGT-3') [3], which target a 207-bp region of VP2, viral sequences were obtained from five wolves and four foxes. BLAST (http://www.ncbi.nlm.nih.gov) and FASTA (http://www.ebi.ac.uk/fasta33) analysis showed that nine strains (GenBank accession nos. MK496165-73) shared 97.9-99.5\% nt sequence identity with each other and displayed the closest identity to canine and feline $\mathrm{BuV}$ strains (97.5-99.0\% nt sequence identity) [3, 7]. In order to obtain additional genetic information about the VP2 capsid gene, three positive samples with viral loads ranging from $10^{3}$ to $10^{4}$ DNA copies $/ 5 \mu \mathrm{l}$ were used as template to amplify viral DNA, using the primer pairs CPPV-1409F/CPPV-1414R and CPPV-165 F/CPPV-1571R [7], which were designed based on alignments of the available sequences of Italian and the Hungarian canine strains (GenBank accession nos. MF198244-6) and were able to amplify two overlapping fragments of the VP2-coding region of $962 \mathrm{nt}$ (CPPV-1409 F/CPPV-1414R) and $1350 \mathrm{nt}$ (CPPV-165 F/CPPV-1571R) in length, respectively (Table 1). Despite several attempts, for one wolf faecal sample (130/Wolf/2015/ITA, GenBank accession no. MK496167), we obtained a sequence that was $500 \mathrm{nt}$ in length, corresponding to the N-terminus of VP2, using a strategy of heminested PCR with external primers CPPV-1409 F/CPPV-1414R and the internal primer CPPV$165 \mathrm{~F}$. Sequence analysis showed that the wolf strain had 99.0-100\% nt and $100 \%$ aa sequence identity to the Italian and Hungarian canine strains (GenBank accession nos. MF198244-6) and to the Italian feline strains (GenBank accession nos. MK030121-3) [3, 7]. Neighbor-joining

Table 1 List of primers used in this study. Nucleotide positions refer to the sequence of the canine protoparvovirus 2 strain ITA/2011/297-15 (GenBank accession no. MF198244

\begin{tabular}{lllll}
\hline Oligonucleotide & Position & Sequence (5' to 3') & Sense & References \\
\hline CCPV-L3 F & $2938-2964$ & TGAACAAGAAATAGACAACATTGTCAT & + & Martella et al., 2018 [3] \\
CCPV-L3 R & $3012-3035$ & AAAGAGCAGTTAGGTCATTGTTGT & - & Martella et al., 2018 [3] \\
CCPV-165 F & $2872-2891$ & CTGGTTTAATCCAGCAGACT & + & Martella et al., 2018 [3] \\
CCPV-371 R & $3060-3079$ & TGAAGACCAAGGTAGTAGGT & - & Martella et al., 2018 [3] \\
CCPV-1409 F & $2411-2432$ & TCATATTCCTGGAGAAACATCA & + & Diakoudi et al., 2019 [7] \\
CCPV-1414 R & $3351-3372$ & ATATGTCTGTTAGATTGCCAGT & - & Diakoudi et al., 2019 [7] \\
CCPV-1571 R & $4201-4219$ & TTATAGAGTAATATTAGGC & - & Diakoudi et al., 2019 [7] \\
\hline
\end{tabular}


phylogenetic analysis was performed with a selection of partial capsid sequences representative of the genus Protoparvovirus. In the resulting trees (Fig. 1A and B), the wolf and fox sequences clustered tightly with the canine and feline strains. We propose that this lineage could constitute a novel species, tentatively named "carnivore protoparvovirus 2".

Domestic and wild canids are known to be exposed to common viral pathogens, including CPV-2, canine adenoviruses (CAdV1 and CAdV2) and canine distemper virus (CDV) [8-11]. Furthermore, the advent of next-generation sequencing has enabled the identification and characterization of several previously unknown viruses that can be transmitted between dogs and wildlife. These examples include canine kobuviruses (CaKoVs) [12-15], circoviruses [16, 17], and sapoviruses [12, 18]. Therefore, we hypothesized that the carnivore protoparvoviruses recently detected in dogs and cats $[3,7]$ could also circulate among wild canids. In this study, using specific primers and a probe, novel parvoviruses were found in wolves and foxes at a rate of $17.1 \%$ and $10.5 \%$, respectively, suggesting that these viruses are
Fig. 1 (A) Phylogenetic tree based on a portion of the VP2 gene $(\sim 500 \mathrm{nt})$ of the wolf strain 130/2015/ITA (GenBank accession no. MK496167). (B) Phylogenetic tree based on a 200-nt region of the VP2 gene of the wolf and fox strains detected in this study (GenBank accession nos. MK496165-73). Both trees were generated using the neighbor-joining method and the Kimura 2-parameter model (MEGA software package, version 3.0), with statistical support provided by bootstrapping with 1000 replicates. The scale bar represents nucleotide substitutions per site. The labels denote the sequences detected in this study

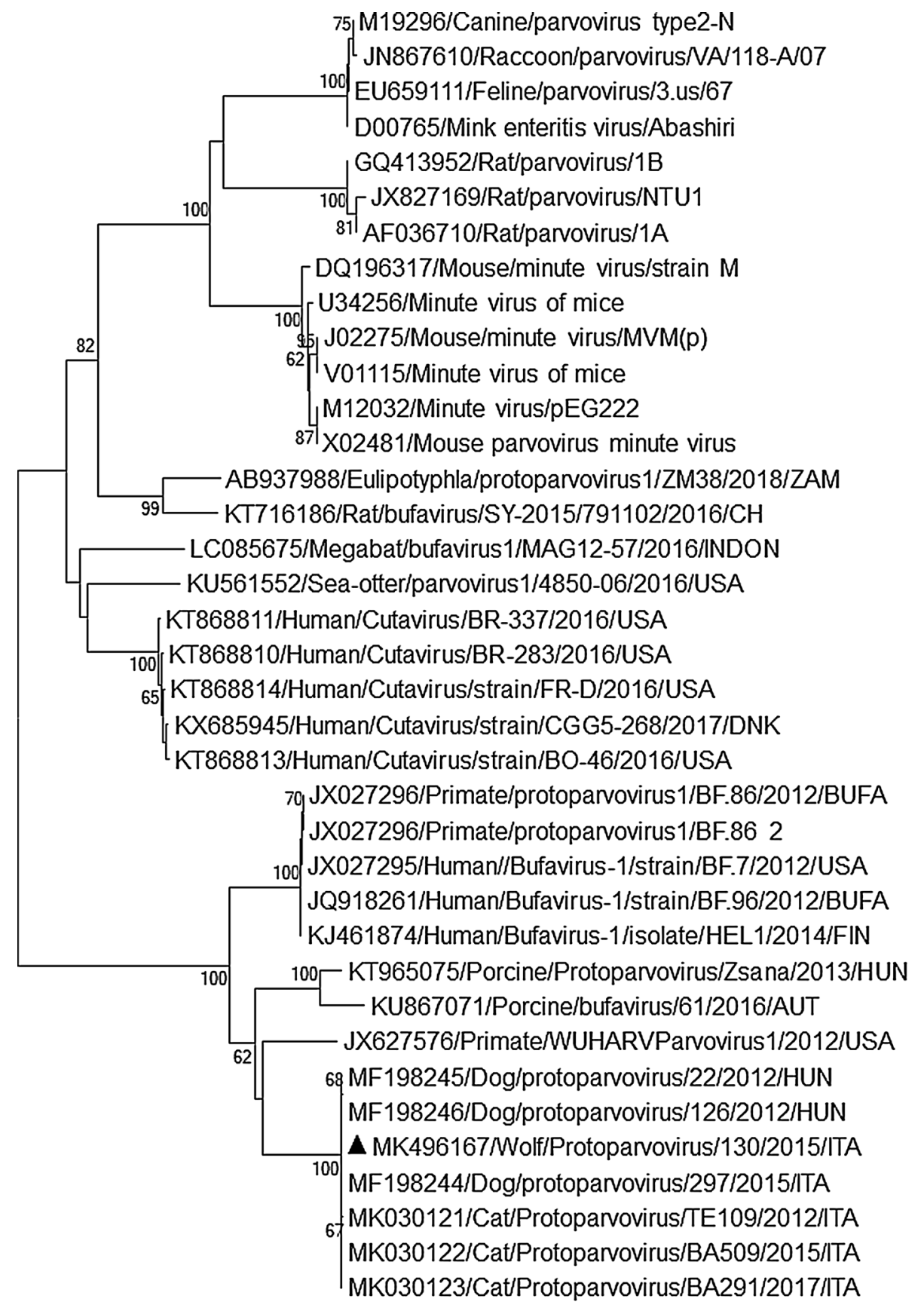

$\longmapsto 0.1$ 
Fig. 1 (continued)

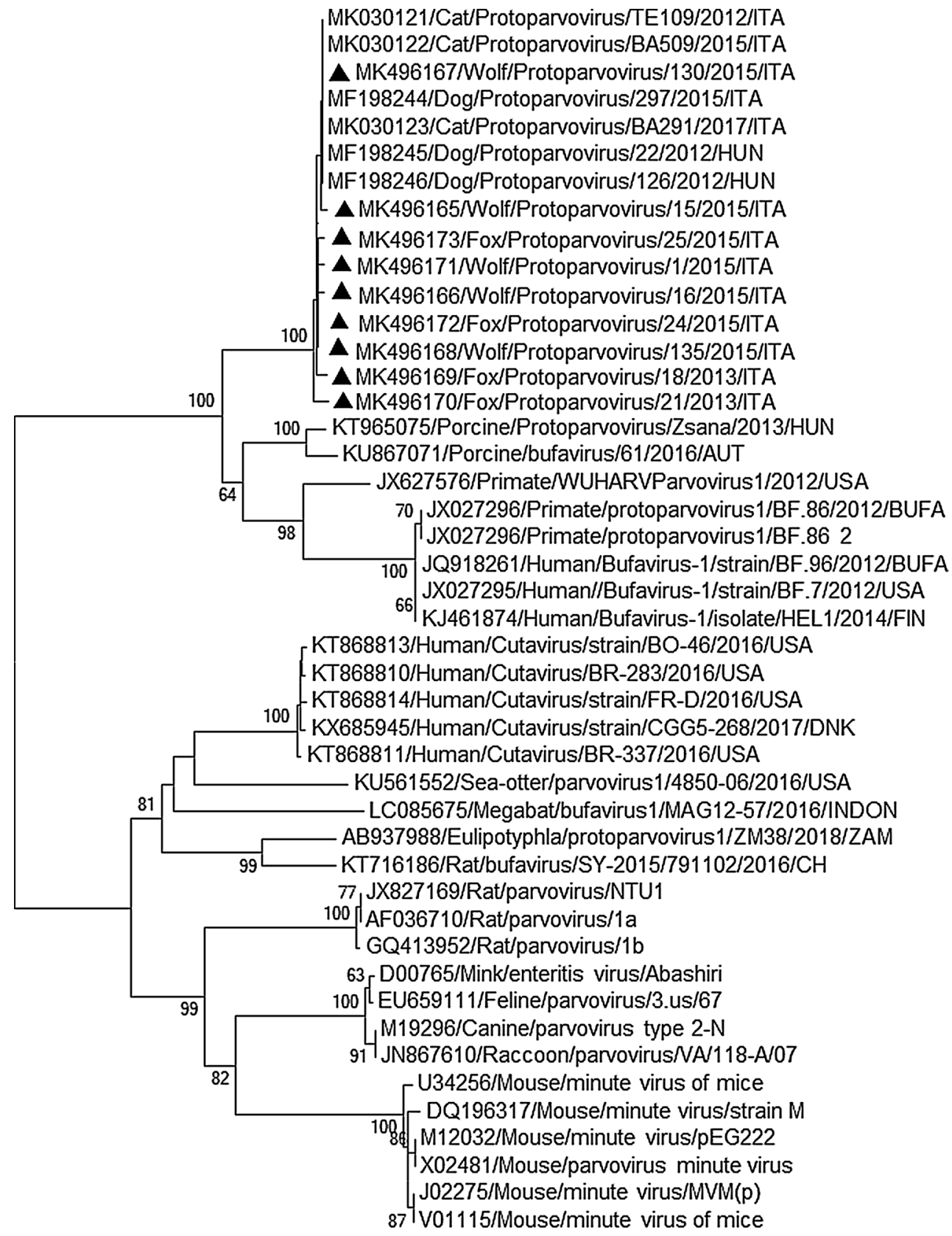

$\stackrel{105}{1}$

probably as common in wild canids as they are in domestic dogs. Analysis of a short fragment (207 nt) of the ORF2 gene, obtained from nine samples, revealed a high degree of genetic relatedness to canine and feline BuVs [3, 7]. This sequence conservation was also found when analyzing a longer region of the capsid gene $(0.5 \mathrm{~kb})$ of the wolf strain 130/Wolf/2015/ITA. However, a more precise and definitive characterization should rely on analysis of the complete genome sequence or at least the full-length capsid gene. In our analysis, all of the positive stool samples were collected from wolves and foxes that were apparently healthy at the time of sampling, and we did not analyze specimens from animals with intestinal lesions or signs. Furthermore, all of the samples analyzed in this study had previously been screened for CPV-2, CadV1 and CadV2, canine coronavirus, $\mathrm{CDV}$, caliciviruses and $\mathrm{CaKoV}[10,14]$, and none of the protoparvovirus-positive animals had mixed infections.

To date, it is unclear whether members of this novel species of parvoviruses play a role in enteric or extra-enteric diseases in carnivores. Molecular screening of canine stool samples from dogs with or without enteritis has revealed that $\mathrm{BuVs}$ are a common component of the faecal virome, 
although there was no significant difference in terms of prevalence between dogs with and without enteric signs. However, screening of canine respiratory samples revealed a positive association between the presence of the virus and respiratory signs [3]. More recently, canine $\mathrm{BuVs}$ that were closely genetically related to those identified in Italian and Hungarian canine samples were detected in serum specimens from dogs in China. Of interest, the positive sera were collected from animals showing signs of CIRD [19]. Conversely, this association was not observed when testing nasal and oropharyngeal swabs of cats with or without signs of URTD [7], although the virus was about 5 to 6 times more common in respiratory samples (9.5\% to $12.2 \%)$ than in diarrhoeic specimens (2.2\%) [7]. Accordingly, it has been hypothesized that these protoparvoviruses may have a preferential tropism for the respiratory tract $[3,7]$. In our study, we were able to identify the virus in the enteric tract of wild animals. It is therefore possible that faecal shedding of $\mathrm{BuVs}$ represents a strategy of virus persistence in animal populations.

In conclusion, the molecular data collected in our study demonstrate that similar BuVs circulate in dogs, cats and wild canids, extending the host range of these BuVs. This finding is intriguing, as it mirrors what has been observed over several decades of investigations for carnivore protoparvovirus $1[8,10,20]$.

Funding This work was financed by grants from Università degli Studi di Teramo.

\section{Compliance with ethical standards}

Conflict of interest All authors declare that there are no financial or other relationships that might lead to a conflict of interest. All authors have seen and approved the manuscript and have contributed significantly to the work.

Ethics standards All applicable international, national, and/or institutional guidelines for the care and use of animals were followed.

\section{References}

1. Berns KI, Parrish CR (2015) Parvoviridae. In: Knipe DM, Howley PM, Cohen JI, Griffin DE, Lamb RA, Martin MA, Racaniello VR, Roizman B (eds) Fields virology, 6th edn. Lippincott Williams \& Wilkins, Philadelphia, pp 1768-1791

2. Cotmore SF, Tattersall P (2005) Structure and organization of the viral genome. In: Kerr J, Cotmore SF, Bloom ME, Linden RM, Parrish CR (eds) Parvoviruses. Hodder Arnold, London, pp 73-94

3. Martella V, Lanave G, Mihalov-Kovács E, Marton S, VargaKugler R, Kaszab E, Di Martino B, Camero M, Decaro N, Buonavoglia C, Bányai K (2018) Novel parvovirus related to primate bufaviruses in dogs. Emerg Infect Dis 24:1061-1068. https://doi. org/10.3201/eid2406.171965
4. Phan TG, Vo NP, Bonkoungou IJ, Kapoor A, Barro N, O'Ryan M, Kapusinszky B, Wang C, Delwart E (2012) Acute diarrhea in West African children: diverse enteric viruses and a novel parvovirus genus. J Virol 86:11024-11030. https://doi.org/10.1128/ JVI.01427-12

5. Yahiro T, Wangchuk S, Tshering K, Bandhari P, Zangmo S, Dorji T, Tshering K, Matsumoto T, Nishizono A, SöderlundVenermo M, Ahmed K (2014) Novel human bufavirus genotype 3 in children with severe diarrhea, Bhutan. Emerg Infect Dis 20:1037-1039. https://doi.org/10.3201/eid2006.131430

6. Handley SA, Thackray LB, Zhao G, Presti R, Miller AD, Droit L, Abbink P, Maxfield LF, Kambal A, Duan E, Stanley K, Kramer J, Macri SC, Permar SR, Schmitz JE, Mansfield K, Brenchley JM, Veazey RS, Stappenbeck TS, Wang D, Barouch DH, Virgin HW (2012) Pathogenic simian immunodeficiency virus infection is associated with expansion of the enteric virome. Cell 151:253-266. https://doi.org/10.1016/j. cell.2012.09.024

7. Diakoudi G, Lanave G, Capozza P, Di Profio F, Melegari I, Di Martino B, Pennisi MG, Elia G, Cavalli A, Tempesta M, Camero M, Buonavoglia C, Bányai K, Martella V (2019) Identification of a novel parvovirus in domestic cats. Vet Microbiol 228:246-251. https://doi.org/10.1016/j.vetmic.2018.12.006

8. Battilani M, Scagliarini A, Tisato E, Turilli C, Jacoboni I, Casadio R, Prosperi S (2001) Analysis of canine parvovirus sequences from wolves and dogs isolated in Italy. J Gen Virol 82:1555-1560. https://doi.org/10.1099/0022-1317-82-7-1555

9. Balboni A, Verin R, Morandi F, Poli A, Prosperi S, Battilani M (2013) Molecular epidemiology of canine adenovirus type 1 and type 2 in free-ranging red foxes (Vulpes vulpes) in Italy. Vet Microbiol 162:551-557. https://doi.org/10.1016/j.vetmi c.2012.11.015

10. Melegari I, Sarchese V, Di Profio F, Robetto S, Carella E, Bermudez Sanchez S, Orusa R, Martella V, Marsilio F, Di Martino B (2018) First molecular identification of kobuviruses in wolves (Canis lupus) in Italy. Arch Virol 163:509-513. https://doi. org/10.1007/s00705-017-3637-1

11. Deem SL, Spelman LH, Yates RA, Montali RJ (2000) Canine distemper in terrestrial carnivores: a review. J Zoo Wildl Med 31:441-451. https://doi.org/10.1638/1042-7260(2000)031\%5b044 1:CDITCA\%5d2.0.CO;2

12. Li L, Pesavento PA, Shan T, Leutenegger CM, Wang C, Delwart E (2011) Viruses in diarrhoeic dogs include novel kobuviruses and sapoviruses. J Gen Virol 92:2534-2541. https://doi.org/10.1099/ vir.0.034611-0

13. Kapoor A, Simmonds P, Dubovi EJ, Qaisar N, Henriquez JA, Medina J, Shields S, Lipkin WI (2011) Characterization of a canine homolog of human Aichivirus. J Virol 85:11520-11525. https://doi.org/10.1128/JVI.05317-11

14. Di Martino B, Di Profio F, Melegari I, Robetto S, Di Felice E, Orusa R, Marsilio F (2014) Molecular evidence of kobuviruses in free-ranging red foxes (Vulpes vulpes). Arch Virol 159:18031806. https://doi.org/10.1007/s00705-014-1975-9

15. Olarte-Castillo XA, Heeger F, Mazzoni CJ, Greenwood AD, Fyumagwa R, Moehlman PD, Hofer H, East ML (2015) Molecular characterization of canine kobuvirus in wild carnivores and the domestic dog in Africa. Virol 477:89-97. https://doi. org/10.1016/j.virol.2015.01.010

16. Kapoor A, Dubovi EJ, Henriquez-Rivera JA, Lipkin WI (2011) Complete genome sequence of the first canine circovirus. J Virol 86:7018. https://doi.org/10.1128/JVI.00791-12

17. Zaccaria G, Malatesta D, Scipioni G, Di Felice E, Campolo M, Casaccia C, Savini G, Di Sabatino D, Lorusso A (2016) Circovirus in domestic and wild carnivores: an important opportunistic agent? Virology 490:69-74. https://doi.org/10.1016/j.virol .2016 .01 .007 
18. Olarte-Castillo XA, Hofer H, Goller KV, Martella V, Moehlman PD, East ML (2016) Divergent sapovirus strains and infection prevalence in wild carnivores in the Serengeti ecosystem: a longterm study. PLoS One 11:e0163548. https://doi.org/10.1371/journ al.pone. 0163548

19. Sun W, Zhang S, Huang H, Wang W, Cao L, Zheng M, Yin Y, Zhang H, Lu H, Jin N (2019) First identification of a novel parvovirus distantly related to human bufavirus from diarrheal dogs in China. Virus Res 19:30048-30056. https://doi.org/10.1016/j. virusres.2019.03.020
20. Truyen U, Evermann JF, Vieler E, Parrish CR (1996) Evolution of canine parvovirus involved loss and gain of feline host range. Virol 215:186-189. https://doi.org/10.1006/viro.1996.0021

Publisher's Note Springer Nature remains neutral with regard to jurisdictional claims in published maps and institutional affiliations. 